\title{
Disjoint Identifying-Codes for Arbitrary Graphs
}

\author{
Moshe Laifenfeld and Ari Trachtenberg \\ Electrical and Computer Engineering \\ Boston University \\ Boston, MA \\ Email: moshel,trachten@bu.edu
}

\begin{abstract}
Identifying codes have been used in a variety of applications, including sensor-based location detection in harsh environments. The sensors used in such applications are typically battery powered making energy conservation an important optimization criterion for lengthening network lifetime. In this work we propose and develop the concept of disjoint identifying codes with the motivation of providing energy load-balancing in such systems. We also provide information-theoretic upper and lower bounds on the number of disjoint identifying codes in a given graph, and show that these bounds are asymptotically tight for a modification of Hadamard matrices.
\end{abstract}

A version of this paper should be presented at the IEEE Symposium on Information on Information Theory 2005.

\section{INTRODUCTION}

Identifying codes have been proposed and implemented for a variety of applications, including fault diagnosis of multiprocessor systems [1] and location detection in harsh environments [2-6]. The latter application is designed for implementation in a network of battery-powered microprocessors (more generally, "sensors").

Specifically, in this approach, a continuous coverage area is quantized into a finite set of regions, and beacon nodes are placed in certain regions according to an identifying code property, meaning that a receiver in any given region will reliably hear a unique (and hence identifying) collection of beacons. In the theoretical model of the problem, regions are represented by vertices in a graph (with beacon nodes being called "codewords") and edges connect vertices whose regions are within reception range.

Since beacon nodes are typically battery-powered, the lifetime of an identifying code-based location detection system is limited by the lifetime of a single beacon node. A reasonable approach to prolonging the lifetime of such a system is to balance beaconing responsibilities among $\kappa$ different identifying codes. If these identifying codes are also disjoint, then the system lifetime is thereby roughly multiplied by a factor $\kappa$. In addition, such a system benefits from a spatial diversity that can be utilized by a user to improve robustness.

In this work, we address the question of how many disjoint identifying codes $\kappa$ are available to an arbitrary location-detecting system. We proceed in Section II with a review of related research and a formal statement of our 
problem. Thereafter, Section III develops some information-theoretic upper bounds on $\kappa$, and Section IV utilizes the symmetric Lovăsz Local Lemma to develop some lower bounds. Finally in Section V we present our conclusions.

\section{Preliminaries}

\section{A. Related work}

A variety of related work sexists in the information theory and theoretical computer science communities.

1) Identifying codes: Intuitively, an identifying code of radius $r$ is a set of vertices in a graph $G$ with the property that any ball of radius $r$ in $G$ has a unique intersection with the identifying code (a technical definition will be given in Section II-B). Identifying codes were initially introduced in [1] with an application to fault diagnosis in multiprocessor systems. In that application, each processor reports to a central point whether they detect a malfunction in one of their neighbors or themselves. By coalescing the various reports, the central point can uniquely determine the faulty processor. Several bounds on the minimum size of an identifying code were also presented in [1] and studied further in [7, 8] and many subsequent works. In [3,4] identifying codes of radius $r=1$ were suggested for use in indoor location detection and thereafter refined for purposes of robustness.

2) Domatic number: One well-known generalization of our work is the problem of determining the domatic number of a graph, which is the largest number of disjoint dominating sets in the graph. Recall that a dominating set is a set of vertices that, together with their neighbors, contain all the vertices of the graph. Not surprisingly, the theoretical tools and applications of dominating sets are similar to those explored in this work. One such application involves replicating resources in a distributed system so that each system member has access to the range of resources types. By cycling resources through disjoint dominating sets in a system graph, one can improve the robustness of the system with respect to connection failures. On the theoretical size, Feige et al. [9] have applied

the Lovăsz Local Lemma (LLL) to approximate the domatic number of an arbitrary graph as $(1-o(1)) \frac{b_{\min }}{\ln \left(b_{\max }-1\right)}$ where $b_{\min }$ and $b_{\max }$ are the minimum and maximum vertex neighborhood ball sizes respectively.

3) Locating-dominating sets: The closest literature to this work involves the problem of locating-dominating sets [10-12]. A set of vertices $S$ is said to be locating-dominating if every vertex not in $S$ is a neighbor of a unique subset of vertices in $S$. As such, these sets are codes that identify all vertices not in the set, a subtle but important difference from standard identifying codes. It has been shown in [11] that whenever there is an identifying code of size $s$ in a graph, there is also a locating-dominating set of size at most $s$.

\section{B. Technical definitions}

Given an directed graph $G$ with vertices $V$ and edges $E$, the incoming ball $B^{+}(v)$ consisting of edges directed towards $v \in V$, together with $v$; likewise, the outgoing ball $B^{-}(v)$ consisting of edges directed away from $v$, together with $v$. For undirected graphs, we shall simply use the notation $B(v)=B^{+}(v)=B^{-}(v)$. We shall correspondingly use $b^{+}(v), b^{-}(v)$, and $n$ to denote the sizes of $B^{+}(v), B^{-}(v)$, and $V$ respectively. 
A non-empty subset $\mathbb{C} \subseteq V$ is called a code and its elements are codewords. For a given code $\mathbb{C}$, the identifying set $I_{\mathbb{C}}(v)$ of a vertex $v$ is defined to be the codewords directed towards $v$, i.e., $I_{\mathbb{C}}(v)=B^{+}(v) \cap \mathbb{C}$. A code $\mathbb{C}$ is an identifying code if each identifying set of the code is unique, in other words

$$
\forall u, v \in V \quad u=v \longleftrightarrow I_{\mathbb{C}}(u)=I_{\mathbb{C}}(v) .
$$

In some applications, as in [3], (though not in this paper) it is desirable to require identifying codes to have only non-empty identifying sets. In this paper, we shall be concerned specifically with the number of vertex-disjoint identifying codes $\kappa(G)$ in a given graph.

\section{UPPER BOUNDS ON $\kappa(G)$}

We next present upper bounds on the number of disjoint identifying codes in a given graph.

\section{A. A trivial bound}

One trivial bound derives from a simple distance metric on identifying sets.

Definition 1 The difference set $D_{\mathbb{C}}(u, v)$ is defined to be the symmetric difference between the identifying sets of vertices $u, v \in V$ :

$$
D_{\mathbb{C}}(u, v)=\left[I_{\mathbb{C}}(u)-I_{\mathbb{C}}(v)\right] \cup\left[I_{\mathbb{C}}(v)-I_{\mathbb{C}}(u)\right],
$$

where subtraction denotes set difference. We shall use $d_{\mathbb{C}}(u, v)$ to denote the size of $D_{\mathbb{C}}(u, v)$.

We shall combine difference sets into a difference set collection $\mathbf{D}_{\mathbb{C}}=\cup_{u, v \in V}\left\{D_{\mathbb{C}}(u, v)\right\}$. Note that $D_{\mathbb{C}}(u, v)=$ $D_{\mathbb{C}}(v, u)$ so that only one of these appears in $\mathbf{D}_{\mathbb{C}}$.

Definition 2 The minimum distance of a code $\mathbb{C}$ is given by $d_{\text {min }}(\mathbb{C})=\min _{u \neq v \in V} d_{\mathbb{C}}(u, v)$. When $\mathbb{C}=V$, we shall denote $d_{\min } \hat{=} d_{\min }(V)$.

The following lemma follows straightforwardly.

Lemma $1 \mathbb{C}$ is an identifying code iff $d_{\min }(\mathbb{C})>0$.

We may now derive the following trivial bound.

Theorem 1 For a given graph $G$,

$$
\kappa(G) \leq d_{\min }
$$

Proof: Suppose the minimum distance of the graph is attained between vertices $u$ and $v$. Lemma 1 then implies that every identifying code must contain at least one element of $d_{V}(u, v)$, meaning that at most $d_{\min }$ disjoint identifying 
codes exist for $G$.

\section{B. Identifying code bound}

We can also bound $\kappa$ using a lower bound on the size of an identifying code.

Theorem 2 For a graph $G$ with $n=|V|$ vertices,

$$
\kappa(G) \leq\left\lfloor\frac{1}{\log (n)} \sum_{v \in V} H\left(\frac{b^{-}(v)}{n}\right)\right\rfloor,
$$

where $H(p)=-p \log p-(1-p) \log (1-p)$ is the binary entropy function and all logarithms have the same base.

Example 1 demonstrates an application of Theorems 1 and 2 to modified Hadamard matrices [13], and Example 2 generalizes this to a family of graphs that asymptotically reach the bounds in this paper. Before proving Theorem 2 , we adapt the following bound on the size of an identifying code from the related result in [1].

Lemma 2 For any identifying code $\mathbb{C}$,

$$
\log n \leq \sum_{c \in \mathbb{C}} H\left(\frac{b^{-}(c)}{n}\right) .
$$

Proof of Lemma 2: Let the random variable $Y$ represent the unique identifier in the range $1 \ldots n$ of a uniformly randomly selected vertex. Furthermore, let $X_{i}$ be 1 if the selected vertex is in $b^{-}\left(c_{i}\right)$, where $c_{i}$ is the $i$-th codeword of $\mathbb{C}$, and 0 otherwise. We may then compute the mutual information

$$
I(Y ; X)=H(Y)-H(Y \mid X)=\log n .
$$

On the other hand,

$$
\begin{aligned}
I(Y ; X) & =H(X)-H(X \mid Y) \\
& \leq \sum_{i=1}^{|\mathbb{C}|} H\left(X_{i}\right)=\sum_{c \in \mathbb{C}} H\left(\frac{b^{-}(c)}{n}\right) .
\end{aligned}
$$

Putting together (2) and (3) proves the lemma.

The proof of Theorem 2 now follows from summing (1) over the largest set $S$ (with size $\kappa(G)$ ) of disjoint identifying codes in $G$ :

$$
\begin{aligned}
\sum_{\mathbb{C} \in S} \log n & \leq \sum_{\mathbb{C} \in S} \sum_{c \in \mathbb{C}} H\left(\frac{b^{-}(c)}{n}\right) \\
& \leq \sum_{v \in V} H\left(\frac{b^{-}(v)}{n}\right) .
\end{aligned}
$$


Example 1 Disjoint identifying codes in a modified Hadamard matrix.

Consider deleting the first row and column of the $8 \times 8$ binary Hadamard matrix, and permuting the resulting rows so that the resulting $7 \times 7$ matrix $H^{*}$ has ones along its diagonal as follows:

$$
H^{*}=\left(\begin{array}{lllllll}
1 & 1 & 0 & 1 & 0 & 0 & 1 \\
0 & 1 & 1 & 1 & 1 & 0 & 0 \\
1 & 0 & 1 & 1 & 0 & 1 & 0 \\
0 & 0 & 0 & 1 & 1 & 1 & 1 \\
1 & 1 & 0 & 0 & 1 & 1 & 0 \\
0 & 1 & 1 & 0 & 0 & 1 & 1 \\
1 & 0 & 1 & 0 & 1 & 0 & 1
\end{array}\right) .
$$

We may view $H^{*}$ as the adjacency matrix of a 7-vertex directed graph $G^{*}$, whose outgoing balls correspond to columns of $H^{*}$ and thus have size 4 . The minimum distance of $G^{*}$ will also be 4 corresponding to the Hamming distance between rows of $H^{*}$. As such, Theorem 1 bounds $\kappa\left(G^{*}\right) \leq 4$ and Theorem 2 bounds $\kappa\left(G^{*}\right) \leq 2.4567$; an exhaustive search shows that there are exactly 2 disjoint identifying codes in the graph.

In the limit, the number of identifying codes is bounded by the number of vertices $n$, the largest outgoing ball $b_{\max }^{-}$ , and the minimum distance $d_{\min }$ of the graph, as the following Corollary shows.

Corollary 1 For sufficiently large graphs,

$$
\kappa \leq\left\{\begin{array}{ll}
\min \left(b_{\max }^{-}, d_{\text {min }}\right) & \text { if } b_{\text {max }}^{-} \text {is } o(n), \\
\min \left(\frac{n}{\log n}, d_{\text {min }}\right) & \text { if } b_{\text {max }}^{-} \text {is } \Theta(n) .
\end{array},\right.
$$

where $o$ and $\Theta$ denote asymptotically comparison (smaller than and equal to respectively).

Proof: Taking Theorem 2 in the limit yields:

$$
\begin{aligned}
\lim _{n \longrightarrow \infty} \kappa & \leq \lim _{n \rightarrow \infty} \frac{n}{\log n} H\left(\frac{b_{\max }^{-}}{n}\right) \\
& =\lim _{n \rightarrow \infty}\left[\begin{array}{c}
b_{\max }^{-}\left(1-\frac{\log \left(b_{\max }^{-}\right)}{\log n}\right)- \\
\frac{n-b_{\max }^{-}}{\log n} \log \left(1-\frac{b_{\max }^{-}}{n}\right)
\end{array}\right] \\
& =b_{\max }^{-}-\lim _{n \longrightarrow \infty}\left[\frac{n}{\log n} \log \left(1-\frac{b_{\max }^{-}}{n}\right)\right] .
\end{aligned}
$$

If $b_{\max }^{-}$is $o(n)$, then (6) reduces to $b_{\max }^{-}$. On the other hand, if $b_{\max }^{-}$is $\Theta(n)$, then (6) becomes $\frac{n}{\log n}$. Applying Theorem 1 completes the proof. 


\section{LOWER BOUNDS ON $\kappa$}

The main results in this section, Theorems 4 and 5, provide lower bounds on $\kappa$ based on the symmetric form of the Lovăsz Local Lemma (LLL) [14], a powerful probabilistic tool for proving the existence of an event. We restate this form of LLL for completeness.

Lemma 3 Consider a set of events $\mathcal{E}=\left\{E_{1} \ldots E_{n}\right\}$ such that $\operatorname{Pr}\left[E_{i}\right] \leq p$ for all $i$. If, for each $i, E_{i}$ is mutually independent of events in any set $F \subseteq \mathcal{E}$ of size $|F|>|\mathcal{E}|-m$, then

$$
e p(m+1) \leq 1 \quad \Rightarrow \quad \operatorname{Pr}\left[\cap_{i=1}^{n} \bar{E}_{i}\right]>0,
$$

where $e$ is the base of the natural logarithm.

\section{A. Translation to LLL}

We first translate the problem of finding disjoint identifying codes into one of assigning vertex colors meeting specific constraints.

Lemma 4 There exist $\kappa$ disjoint identifying codes in a graph $G=(V, E)$ if and only if $V$ can be colored with $\kappa$ colors so that $D_{V}(u, v)$ contains at least one vertex of each color for all $u \neq v \in V$.

The key to establishing a lower bound will thus be to find the threshold value $\kappa$ for which a random $\kappa$-coloring of $V$ will, with some non-zero probability, provide at least one vertex of each color in every difference set. We shall accomplish this by means of symmetric LLL.

As such, define an event $E_{i,(u, v)}$ to denote the case when the $i$-th color does not appear among the first $d_{\text {min }}$ vertices of $D_{V}(u, v)$ under a random, uniform and independent $\kappa$-coloring of $V$. Clearly,

$$
p \hat{=} \operatorname{Pr}\left[E_{i,(u, v)}\right]=\left(1-\frac{1}{\kappa}\right)^{d_{\min }} \leq e^{\frac{d_{\min }}{\kappa}} .
$$

We next bound the number of mutual dependencies between events in $\mathcal{E}$ using the fact that $v$ only appears in difference sets of vertex pairs where one vertex is in $B^{-}(v)$ and the other is not.

Lemma 5 Each vertex $v \in V$ appears in exactly

$$
b^{-}(v)\left(n-b^{-}(v)\right)
$$

difference sets in $\mathbf{D}_{\mathbb{C}}$.

Observe that (8) reaches a maximum at $b^{-}(v)=\left\lfloor\frac{n}{2}\right\rfloor$. Thus, the vertex whose outgoing ball has size closest to $\left\lfloor\frac{n}{2}\right\rfloor$ will appear in the largest number of difference sets and thus can be said to be the most distinguishing. We shall denote the size of this most distinguishing ball by $\tilde{b}$. 
Example 2 A family of codes where Theorems 2 and 4 meet asymptotically.

Consider the generalization of Example 1 to a $2^{n}-1 \times 2^{n}-1$ matrix $H_{n}^{*}$ resulting from similarly modifying the $2^{n} \times 2^{n}$ Hadamard matrix (i.e., deleting the first row and column and permuting the remaining rows to give ones on the diagonal). By definition of the Hadamard matrix, $H_{n}^{*}$ will have outgoing ball size and minimum distance equal to $2^{n-1}$. Thus, Theorems 2 and 4 imply that that the corresponding graph $G_{n}^{*}$ has

$$
\frac{2^{n-1}}{2 \ln \left[2^{n-1}\left(2^{n}-2^{n-1}-1\right)\right]} \leq \kappa\left(G_{n}^{*}\right) \leq \frac{\left(2^{n}-1\right) H\left(\frac{2^{n-1}}{2^{n}-1}\right)}{\log \left(2^{n}-1\right)} .
$$

As $n$ approaches infinity:

$$
\left(\frac{1}{4 \ln 2}\right) \frac{2^{n}}{n} \leq \kappa\left(G_{\infty}^{*}\right) \leq \frac{2^{n}}{n},
$$

indicating that the upper and lower bounds asymptotically converge.

Theorem 3 For a given graph $G=(V, E)$ with $n$ vertices,

$$
d_{\min } \leq \frac{2 \tilde{b}(n-\tilde{b})}{n-1} .
$$

Proof: Applying Lemma 5 to the sum

$$
S=\sum_{D_{V}(u, v) \in \mathbf{D}_{\mathbb{C}}} d_{v}(u, v)
$$

results in $S \leq n \tilde{b}(n-\tilde{b})$. On the other hand, we know that $d_{v}(u, v) \geq d_{\min }$. Putting these facts together proves the lemma.

\section{B. The main bounds}

We now present the first lower bound on the number of disjoint identifying codes in a graph.

Theorem 4 For any non-trivial ${ }^{1}$ connected graph $G$ with $n \geq 6$ vertices,

$$
\kappa(G) \geq \frac{d_{\min }}{2 \ln (\tilde{b}(n-\tilde{b}))} .
$$

Proof: Consider coloring the vertices of $G$ uniformly at random with

$$
k=\frac{d_{\min }}{2 \ln (\tilde{b}(n-\tilde{b}))} \text { colors. }
$$

Then the event $E_{i,(u, v)}$ defined in Section IV-A is dependent on all events $E_{k,\left(u^{\prime}, v^{\prime}\right)}$ where $D_{V}(u, v)$ and $D_{V}\left(u^{\prime}, v^{\prime}\right)$ have their first $d_{\min }$ vertices in common. There are at most

$$
m=k d_{\min } \tilde{b}(n-\tilde{b})
$$

\footnotetext{
${ }^{1}$ We define trivial graphs to include only complete graphs and graphs with no edges, both of which render the theorem meaningless.
} 
such dependent events.

Putting together (10) and (11), we see that

$$
\begin{aligned}
e p(m+1) & \leq e^{\frac{d_{\min }}{k}+1}\left(k d_{\min } \tilde{b}(n-\tilde{b})+1\right) \\
& =\left(\frac{e d_{\min }^{2}}{2 \tilde{b}(n-\tilde{b}) \ln (\tilde{b}(n-\tilde{b}))}\right)+\frac{e}{\tilde{b}^{2}(n-\tilde{b})^{2}} \\
& \leq d_{\min }\left(\frac{e}{(n-1) \ln (2 n-4)}\right)+\frac{e}{4(n-2)^{2}} \\
& \leq{ }_{n \geq 6} 1 .
\end{aligned}
$$

Thus, we may apply symmetric LLL to see that some $k$-coloring will have the property that every color will be present in every difference set. Applying Lemma 4 then completes the proof.

Example 2 shows that Theorem 4 is asymptotically tight for certain graphs. It is also possible to bound $\kappa(G)$ in terms of the minimum and maximum degrees of vertices in $G$, independently of $n$. We provide the theorem statement here without its rather involved proof due of space considerations. It is based on the the maximum size of a directed ball of radius $2, b_{2, \mathrm{MAX}}$, and the outgoing ball size $\tilde{b_{2}}$ closest to $\frac{b_{2, \mathrm{MAX}}}{2}$.

Theorem 5 For any non-trivial graph $G$ with $b_{2, M A X} \geq 9$,

$$
\kappa(G) \geq \frac{\delta}{2 \ln \left(\tilde{b_{2}}\left(b_{2, M A X}-\tilde{b_{2}}+1\right)\right)},
$$

where $\delta=\min \left(d_{\min }, b_{\min }^{+}\right)$, and $b_{\min }^{+}=\min _{v \in V}\left(b^{+}(v)\right)$.

Roughly speaking, Theorem 5 replaces $n$ and $\tilde{b}$ of Theorem 4 with $b_{2, \operatorname{MAX}}$ and $\tilde{b_{2}}$, providing a better bound for some topologies. Example 3 gives one example where Theorem 5 provides a better bound than Theorem 4 .

\section{Conclusions}

In this paper we determined bounds on the number of disjoint identifying codes in an arbitrary non-trivial, connected graph, motivated by applications to energy balancing in location detection systems. Unlike classical identifying codes, we considered both directed and undirected graphs, permitting applications with asymmetric communication channels.

Our results show that the minimum distance between identifying sets of a graph is a key determiner of the number of disjoint identifying codes the graph will allow. Roughly speaking, graphs with larger minimum distances have more disjoint codes, although the sizes of ball neighborhoods of radius 1 and 2 around the vertices of a graph also are important.

We also showed that our bounds are tight for certain graphs based on Hadamard matrices by demonstrating that our lower and upper bounds approach within a small multiplicative constant for these cases. A different lower bound 
Example 3 A family of codes where the bound in Theorem 5 is tighter than that of Theorem 4.

Consider replacing the one in the 7-th row and 1-st column of $H^{*}$ of Example 1 by a zero, resulting in a matrix $H^{1}$. Furthermore, denote by $\mathbf{0}$ the $7 \times 7$ zero matrix and by $\mathbf{0}^{1}$ the the matrix of consisting of a single one in the 7-th column and 1-st row (and zeroes elsewhere). We then construct the following $7 \mathrm{~m} \times 7 \mathrm{~m}$ block Toeplitz adjacency matrix:

$$
H^{m}=\left(\begin{array}{ccccc}
H^{1} & \mathbf{0} & \cdots & \mathbf{0} & \mathbf{0}^{1} \\
\mathbf{0}^{1} & H^{1} & \mathbf{0} & \cdots & \mathbf{0} \\
\mathbf{0} & \mathbf{0}^{1} & H^{1} & \ddots & \vdots \\
\vdots & \ddots & \ddots & \ddots & \mathbf{0} \\
\mathbf{0} & \cdots & \mathbf{0} & \mathbf{0}^{1} & H^{1}
\end{array}\right) .
$$

Note that $H^{m}$ has the same values for $d_{\min }, b^{+}(v)$, and $b^{-}(v)$ as the original $H^{*}$ graph, and that $b_{2, \operatorname{MAX}}=10$. Applying Theorem 5 to the corresponding graph $G^{m}$ results in $\kappa\left(G^{m}\right)>0.6$ for all $m$, whereas Theorem 4 approaches 0 as $m$ approaches infinity. In the more general case we can replace $H^{1}$ by a similar $2^{n}-1 \times 2^{n}-1$ matrix generated from the $2^{n} \times 2^{n}$ Hadamard matrix. In this case, Theorem 5 yields

$$
\kappa\left(G_{n}^{m}\right) \geq \frac{2^{n-2}}{\ln \left(2^{n-1}\left(2^{n}-1\right)\right)}
$$

and as $n$ approaches infinity

$$
\kappa\left(G_{\infty}^{m}\right) \geq \frac{1}{8 \ln 2} \frac{2^{n}}{n}
$$

was provided that is independent of the graph size, and it was shown to be tighter on a block Toeplitz generalization of Hadamard matrices.

Unfortunately, our bounds are clearly not tight for other types of graphs, especially those with low minimum distance. We hypothesize that our bounds can be improved by applying the more general asymmetric form of the Lovăsz Local Lemma, and by introducing more elaborate descriptive graph parameters, such as the minimum distance between balls of radius 2 . On the algorithmic side, we have devised and implemented an efficient greedy algorithm for generating identifying codes (though it is not presented herein). When executed iteratively this algorithm generates disjoint identifying code sets that are generally close to the upper bound on the paper, although string performance guarantees have yet to be proven. One could consider derandomization of the symmetric LLL as another approach for generating such codes.

\section{ACKNOWLEDGMENT}

We would like to thank David Starobinski for originally motivating the disjoint ID-code problem. This work was supported in part by the US National Science Foundation under grants CAREER CCR-0133521, ANI-0240333, and CNS-0435312. 


\section{REFERENCES}

[1] Mark G. Karpovsky, Krishnendu Chakrabarty, and Lev B. Levitin, "A new class of codes for identification of vertices in graphs," IEEE Transactions on Information Theory, vol. 44, no. 2, pp. 599-611, March 1998.

[2] R. Ungrangsi, "Location detection in emergency sensor networks using robust identifying codes," M.S. thesis, Boston University, 2003.

[3] S. Ray, D. Starobinski, A. Trachtenberg, and R. Ungrangsi, "Robust location detection with sensor networks," IEEE Journal on Selected Areas in Communications (Special Issue on Fundamental Performance Limits of Wireless Sensor Networks), vol. 22, no. 6, August 2004.

[4] S. Ray, R. Ungrangsi, F. De Pellegrinin, A. Trachtenberg, and D. Starobinski, "Robust location detection in emergency sensor networks," Proc. INFOCOM, April 2003.

[5] Krishnendu Chakrabarty, Hairong Qi, Sitharama S. Iyengar, and Eungchun Cho, "Coding theory framework for target location in distributed sensor networks," in International Symposium on Information Technology: Coding and Computing, 2001, pp. 130 -134.

[6] R. Ungrangsi, A. Trachtenberg, and D. Starobinski, "An implementation of indoor location detection systems based on identifying codes," Proc. of the IFIP International Conference on Intelligence in Communication Systems (INTELLCOMM 04), 2004.

[7] I. Honkala and A. Lobstein, "On identifying codes in binary hamming spaces," Journal of Comb. Theory, Series A, vol. 99, no. 2, pp. 232-243, 2002.

[8] U. Blass, I. Honkala, and S. Litsyn, "Bounds on identifying codes," Discrete Math., vol. 241, no. 1-3, 2001, PLEASE FIX!!!

[9] U. Feige, M. M. Halldorsson, G. Kortsarz, and A. Srinivasan, “Approximating the domatic number," SIAM J. Comput., vol. 32, no. 1, January 2003.

[10] T. W. Haynes, S. T. Hedetniemi, and P. J. Slater, Fundamentals of Domination in Graphs, Marcel Dekker, 1998.

[11] P. J. Slater, "Fault tolerant locating-dominating sets," Discrete Mathematics, vol. 249, no. 1-3, pp. 179-189, 2002.

[12] I. Honkala, T. Laihonen, and S. Ranto, "On location-dominating codes in binary hamming spaces," Discrete Mathematics and Theoretical Computer Science, vol. 6, no. 2, pp. 265-282, June 2004.

[13] F.J. MacWilliams and N.J.A. Sloane, The Theory of Error-Correcting Codes, North-Holland Publishing Company, New York, 1977.

[14] N. Alon and J. Spencer, "The probabilistic method," 1992. 